\title{
Bounds on the energy densities of ground states on static spacetimes of compact objects
}

\author{
Piotr Mareck: \\ Wyzsza Szkola Informatyki i Zarzadzania, Bielsko-Biala, Poland
}

(Dated: January 5, 2019)

\begin{abstract}
In this paper we investigate quantum fields propagating on given, static, spherically symmetric spacetimes, which are isometric to a part of the Schwarzschild spacetime. Without specifying the internal geometry we show, that there exist bounds on the energy densities of ground states of a quantum scalar field on such spacetimes. The bounds (from above and below) come from the socalled Quantum Energy Inequalities, and are centered around the energy density of the Boulware state (the ground state for Schwarzschild spacetime). The specific value of the bound from below depends critically on the distance $\ell$ from the horizon, where the spacetimes of compact objects cease to be isometric to the Schwarzschild spacetime. In the limit of small $\ell$ we prove, that the energy densities of ground states cannot be below the Boulware level.
\end{abstract}

* piotrm@wsi.edu.pl 


\section{INTRODUCTION}

Quantum fields living on a given spacetime can violate the classical energy conditions, in particular, some states can exhibit negative energy densities. However, energy density operators (which are classically non-linear in fields) need to be constructed in a local manner 1], which makes them invariant to the changes of geometry outside of the region of their support. This means, that the two-point functions of quantum states cannot be employed in the definition of pointwise products of fields, as is the case for normal-ordering. Thus, even the "vacuum states" exhibit non-zero expectation values of the energy-density operator. On a generic globally hyperbolic spacetime there exists at present no condition to distinguish a preferred vacuum state, apart from the necessity for it to be a Hadamard state (only for these states we know a systematic construction of operators non-linear in fields). For static spacetimes, however, ground states are distinguished and acceptable, at least if infrared problems do not prohibit their existence. The energy densities of ground states have been calculated, particularly for the spacetimes possessing horizons (eg. the Schwarzschild spacetime) or time-like boundaries [2, 3]. Generally, these densities tend to infinity (in absolute) if the proper distance form the boundary, denoted here by $L$, approaches zero. For spacetimes without boundaries/horizons the densities are everywhere finite (because the ground states are Hadamard states [4]), although it is difficult to obtain concrete values of these important quantities. In this paper we propose a method to put upper and lower bounds on the energy densities of ground states for spacetimes of compact objects. The geometry of these spacetimes is assumed to be horizon-free and isometric to the Schwarzschild geometry for points at the proper distance $\ell$ and larger from the Schwarzschild horizon. In other words: we consider a class of spacetimes parameterized by $\ell$, but leave the internal geometry of these spacetimes unspecified. We will employ Quantum Energy Inequalities [5, 6] to derive the bounds on the energy densities of ground states. Let us introduce the QEI, as is usually done, on the example of massless fileds in the Minkowski spacetime. In this case the energy density of the ground state (the Poincare-invariant vacuum) is zero, and for an arbitrary state $\psi$ the QEI gives

$$
\langle\varrho\rangle_{\psi} \geqslant-\frac{3}{32 \pi^{2} T^{4}}
$$


for an energy density operator smeared in time with the Lorentzian test function

$$
w^{2}(t)=\frac{T}{\pi\left(T^{2}+t^{2}\right)},
$$

(the reason for the notation will later become clear). If we interpret $T$ as the characteristic time scale of measurement, we van infere from (11), that periods of negative energy density fulfill a uncertainty-like inequality restricting their duration and magnitude. Quantum energy inequalities by now have become a reliable tool in quantum field theory: they have been proved for arbitrary test functions [7], also on curved spacetimes with respect to arbitrary reference Hadamard states (difference quantum inequalities of [8]). Also, these results are not specific to scalar fields: there are corresponding QEIs for the Dirac (eg. [9, 10]) and electromagnetic (eg. [6, 11]) fields.

In this paper we follow a line of thought proposed by Fewster and Pfenning [12], namely, for static spacetimes isometric in a certain causally complete region: on the one hand we can identify the local observables supported in this region ([1]), and on the other we can restrict the ground states of both spacetimes to this region. By developing QEIs with either of these two states as the reference state we obtain two inequalities which are necessarily fulfilled by the difference of the energy densities of these states. Thus, if the energy-density of one of these states is known, the other will be bounded from above and below by the QEIs. In section III.1 we make an important geometrical observation, which tells us that although for static observers located in the exterior Schwarzschild region there exists an upper bound on the maximal sampling time $T$, its value can still become very large if the compact object is described by Schwarzschild geometry up to a very small distance from the Schwarzschild horizon. Therefore, even for small distances $L$ from the horizon there can still be long sampling times $T$, provided that $\ell$ is small enough.

The paper is organized as follows: the second section contains all preliminaries necessary for a treatment of massless quantum fields in static spacetimes, as well as the appropriate version of quantum energy inequalities. In the third section we describe briefly the geometry of spacetimes under consideration, with special emphasis on the maximal size of causally complete regions (double-cones) which can be fit into the region isometric to the Schwarzschild spacetime. The fourth section is the main section of this paper. It contains a description of the method which we have outlined above, as well as its application to the problem of finding bounds on the energy density of ground states of compact objects. We 
develop bounds from below and above separately. In the former case, we prove a theorem (theorem 1) which restricts the energy density of the ground state from below (by anchoring it against the energy-density of the Boulware state). In the limit of very small $\ell$ this bound effectively tells us, that the energy density of ground states cannot be much lower than that of the Boulware state. Our results on the bound from above are weaker: we prove, that a bound form above on the energy density of ground states exists, although we cannot guarantee at present, that this density cannot be much higher than that of Boulware state in the limit of very small $\ell$ (i.e. if the compact objects "tend to" a black hole). It should be stressed, that the Boulware state appears here only as a technical tool (convenient, because the two-point function and the energy density for this state are known), employed in the derivation of bounds on the energy density of ground states of spacetimes without horizons.

Obviously, all these results are valid only if the ground states considered here exist. This is the case at least for spacetimes fulfilling the condition (B3) of appendix B.

\section{QUANTUM FIELDS IN CURVED SPACETIMES AND QUANTUM WEAK ENERGY INEQUALITIES}

\section{A. Preliminaries}

In this paper we use the Landau-Lifshitz time-like convention for the metric, the Riemann and the energy-momentum tensors [34]. We will consider static, spherically symmetric spacetimes, with the metric:

$$
d s^{2}=f(r) d t^{2}-\frac{d r^{2}}{h(r)}-r^{2}\left(d \theta^{2}+\sin ^{2} \theta d \phi\right) .
$$

We will consider (globally hyperbolic) spacetimes of compact objects, for which $r \in[0, \infty)$ and $f(r), h(r)$ are strictly positive. Additionally, we will also consider the Schwarzschild spacetime, where $r \in\left(r_{s}, \infty\right), f(r)=\left(1-r_{s} / r\right)=h(r)$. $\left(r_{s}\right.$ stands here for the Schwarzschild radius $r_{s}=2 M$.) We will frequently use the abbreviation $g(r)=\sqrt{f h}$. The surfaces of constant $t$ are Cauchy surfaces for spacetimes which we consider; these surfaces will be denoted by $\Sigma_{t}$.

We will now review the standard structure [13], adapted to the spacetimes we consider, associated with the evolution of classical scalar field $\phi$. Let $S$ denote the space of real initial 
data, compactly supported on $\Sigma_{t}$ :

$$
S=\mathcal{C}_{0}^{\infty}\left(\Sigma_{t}\right) \oplus \mathcal{C}_{0}^{\infty}\left(\Sigma_{t}\right)
$$

The minimally coupled, massless scalar field, fulfills the equation

$$
\square \phi(x)=0,
$$

which can be rewritten in the form

$$
\frac{\partial^{2} \phi}{\partial t^{2}}=-A \phi
$$

In our case

$$
-A u=-f \nabla^{i} \nabla_{i} u=\frac{g}{r^{2}} \partial_{r}\left[g r^{2} \partial_{r} u\right]+\frac{f \hat{L}^{2}}{r^{2}} u, \quad \text { for } u \in \mathcal{D}(A),
$$

where $\nabla^{i}$ denotes the $4 \mathrm{D}$ covariant derivative taken for spatial indices $i=(r, \theta, \phi)$ and $\hat{L}^{2}$ stands, as usual, for the squared angular momentum operator. Introducing the ReggeWheeler-like coordinate " $x$ ", $\partial_{x}=g \partial_{r}$, we make use of the identity

$$
\frac{g}{r^{2}} \partial_{r}\left[g r^{2} \partial_{r} u\right]=\frac{1}{r}\left[\partial_{x}^{2}(r u)-g\left(\partial_{r} g\right) u\right]
$$

Consequently, the operator $A$ acquires the form

$$
A(r u)=\frac{1}{r}\left[-\partial_{x}^{2}+\frac{g \partial_{r} g}{r}-\frac{f \hat{L}^{2}}{r^{2}}\right](r u),
$$

$A$ is initially defined on the dense domain of smooth functions compactly supported on the Cauchy surface (these space of functions will be denoted by $\mathcal{C}_{0}^{\infty}\left(\Sigma_{t}\right)$ ), on which we introduce the scalar product

$$
\left(u_{1}, u_{2}\right)=\int_{\Sigma_{i}} \overline{u_{1}(\vec{x})} u_{2}(\vec{x}) \frac{r^{2} d r d \Omega}{g(r)},
$$

which is the natural (Lebesgue) measure 35] on $\Sigma_{t}$. (Here $d \Omega$ denotes the volume of the 2-sphere.) The complex Hilbert space of square integrable functions w.r.t. the above scalar product will be denoted by $\mathcal{H}$. This space is often called the one-particle Hilbert space.

We will investigate only these spacetimes, where $A$ is a positive operator (see appendix B); in this case there exists a square root of $A$, the 'one-particle Hamiltonian', denoted by h. The dynamics of classical fields, and the construction of ground-state representation of the quantum field algebra for such spacetimes is standard [13, 14]; we will recall the most important features of it for the convenience of the reader. 
Let $\Phi=\left(v, v_{t}\right) \in S$ denote some arbitrary initial data. Then the transformation $\mathcal{V}(t)$ : $\Phi \rightarrow \Phi(t)$

$$
\begin{aligned}
v(t) & =\cos (\mathrm{h} t) v+\sin (\mathrm{h} t) \mathrm{h}^{-1} v_{t} \\
v_{t}(t) & =-\sin (\mathrm{h} t) \mathrm{h} v+\cos (\mathrm{h} t) v_{t}
\end{aligned}
$$

describes the temporal evolution of these data. The symplectic form

$$
\sigma\left(\Phi_{1}, \Phi_{2}\right)=\int_{\Sigma_{t}}\left[\Phi_{1} \nabla_{a} \Phi_{2}-\left(\nabla_{a} \Phi_{1}\right) \Phi_{2}\right] n^{a} d \eta=\int_{\Sigma_{t}}\left(v_{1} v_{t 2}-v_{t 1} v_{2}\right) \frac{r^{2} d r d \Omega}{g(r)}
$$

(where the unit vector $n^{a}$ is the normalized version of $\left(\partial_{t}\right)^{a}$, and $d \eta$ is the volume element on $\left.\Sigma_{t}\right)$ is preserved by $\mathcal{V}(t)$

$$
\sigma\left(\mathcal{V}(t) \Phi_{1}, \mathcal{V}(t) \Phi_{2}\right)=\sigma\left(\Phi_{1}, \Phi_{2}\right)
$$

In order to construct the ground state, one introduces an operator $k: S \rightarrow \mathcal{H}$, which extracts the positive frequency part of the Cauchy data; for $\left[v, v_{t}\right] \in S$ :

$$
k\left[v, v_{t}\right]=\frac{1}{\sqrt{2}}\left[i \sqrt{h} v+\frac{1}{\sqrt{h}} v_{t}\right]
$$

The classical structure, that is the one-particle structure, $\left(\mathcal{H}, k, e^{i \mathrm{ht}}\right)$, and the symplectic space $(S, \sigma, \mathcal{V}(t))$ can be quantized in the usual manner: one constructs the standard Fock space, and introduces the creation and annihilation operators, $a, a^{*}$. In this way the groundstate representation of the algebra of free fields is obtained: for a real test function $\chi$ the smeared field operator is obtained from

$$
\varphi(\chi)=a(k[0, \chi])+a^{*}(k[0, \chi])
$$

and its time derivative from

$$
\dot{\varphi}(\chi)=a(k[\chi, 0])+a^{*}(k[\chi, 0]) .
$$

Some contact can be made with the more common expressions, namely, according to the spectral theorem, there will be a spectral measure associated with the (self-adjoint) operator $A, d \mu_{I}$, which allows for a functional calculus w.r.t. $A$ ( $I$ will index the generalized eigenfunctions of $A$ (the modes), denoted by $F_{I}(\vec{x})$, corresponding to the eigenvalues $\omega_{I}$ (the frequencies)). Than we may express the operator-valued distribution $\varphi(t, \vec{x})$ as

$$
\varphi(t, \vec{x})=\int d \mu_{I} \frac{1}{\sqrt{2 p}}\left[e^{i \omega_{I} t} F_{I}(\vec{x}) a_{I}+e^{-i \omega_{I} t} \bar{F}_{I}(\vec{x}) a_{I}^{*}\right]
$$




\section{B. QEI for static spacetimes}

Here we shall review some of the results of [7], where Quantum Energy Inequalities have been proved for scalar quantum fields propagating on static spacetimes.

Consider a static observer, located at the spatial position $\vec{x}$, whose (normalized) tangent vector is $u^{a}$. Denote by $\rho(w, \vec{x})$ the (smeared) energy density operator which, we assume, has been constructed in a local and generally covariant manner[1], with the help of the point-splitting procedure employing a local Hadamard parametrix. The smearing is such, that it corresponds to a measurement performed by the static observer, sampled in time by a smooth, non-negative function $w^{2}(t)$ :

$$
\rho(w, \vec{x}) \doteq \int d t T_{a b}(\vec{x}, t) u^{a} u^{b} w^{2}(t)
$$

The sampling function is normalized as a probability density:

$$
\int d t w^{2}(t)=1
$$

Fewster and Teo have shown [7], that the energy-density operator, $\varrho(w, \vec{x})$, necessarily fulfills the inequality:

$$
\langle\varrho(w, \vec{x})\rangle_{\psi}-\langle\varrho(w, \vec{x})\rangle_{G} \geqslant Q_{G}[w, \vec{x}]
$$

where $G$ is the ground state, $\psi$ any other state in the folium of $G$ (i.e. $\psi$ need not be pure, and can be prescribed by an arbitrary 'density matrix'), and

$$
Q_{G}[w, \vec{x}] \doteq-\frac{1}{\pi} \int_{0}^{\infty} d \omega \int \frac{d \mu_{I}}{2 \omega_{I}}\left(\frac{\omega_{I}^{2}}{f(t)}-\frac{1}{4} \nabla_{i} \nabla^{i}\right)\left|F_{I}(\vec{x})\right|^{2} \cdot g\left(\omega+\omega_{I}\right) .
$$

(We hope $g(\omega)$ will not be confused with $g(r)$ which parameterizes the metric.) The inequality holds for all smooth compactly supported sampling functions [36] $w(t)$, which are related to $g(\omega)$, by $g(\omega)=|\widehat{w}(\omega)|^{2}$ (the 'hat' stands for the Fourier transform), that is

$$
g(\omega)=\left|\int_{-\infty}^{\infty} d t w(t) e^{-i \omega t}\right|^{2}
$$

Note, that this function is rapidly decaying for large $\omega$, because $w(t)$ is smooth and compactly supported.

We emphasize, that the right-hand-side of the inequality (21) (denoted in the sequel by $Q_{G}[w, \vec{x}]$, because it is a functional of $w$, constructed relatively to the ground state $G$ ) is negative [37] and finite whenever the ground state $G$ exists. This expresses the basic 
message of QEIs, that there is a meaningful restriction on the sub-ground energy density, which depends on $G$ and $w(t)$ alone, for all states of the quantum field.

We will now adapt the QEI to the spherically symmetric case. The angular dependence of the generalized eigenfunctions of $A$ can be separated in the usual way,

$$
F_{I}(\vec{x})=\frac{1}{\sqrt{2 \pi}} \mathrm{R}_{l \omega}(r) Y_{l m}(\theta, \phi) .
$$

which with the help of

$$
\sum_{m=-l}^{l}\left|Y_{l m}\right|^{2}=(2 l+1) / 4 \pi
$$

leads to a further simplification:

$$
Q_{G}[w, \vec{x}]=-\frac{1}{4 \pi^{2} f(r)} \int_{0}^{\infty} d \omega \int_{0}^{\infty} \frac{d p}{p}\left(p^{2}-\frac{A}{4}\right)\left[\sum_{l=0}^{\infty}(2 l+1)\left|\mathrm{R}_{l p}(r)\right|^{2}\right] \cdot g(\omega+p),
$$

We stress, that the differential operator in the round brackets is such, that the integrand is point-wise positive, for all $r, \omega$ and $p$. The square bracket contains what is usually called "a mode sum". This sum is typically difficult to evaluate, even approximately, in concrete models. However, as we shall see in the case of "long measurements" (specified below) only the low-frequency behavior of this sum will be relevant. In some cases, notably in the case of the Schwarzschild spacetime in the region close to the horizon, this behavior is known explicitly. In section IV we will use (26) to derive upper and lower bounds on $\langle\varrho(r)\rangle_{G}$, for the ground state $G$ on the spacetime of a compact object. For such spacetimes, it would require a careful analysis to calculate the mode sums for arbitrary $\omega$ [38]. Nonetheless, general results settle the $\omega$-dependence of these sums at small frequencies (see appendix [B]), and these will employed to derive bounds on the energy density $\langle\varrho(r)\rangle_{G}$.

\section{GEOMETRIC OBSERVATION}

We will now specify the geometry under consideration. Let us take a Cauchy surface of constant $t$ in the Schwarzschild spacetime and draw a sphere at the geodesic distance $\ell$ from the horizon. The interior of this sphere will be filled with matter of some sort (we do not wish to make any choice in this paper, but note that spacetimes of this sort have been constructed [15, 16]). The situation with very small $\ell$ will be of particular interest for us, as it corresponds to a static spacetime which is "on the verge" of collapse. We will 
also consider a static observer, located at the proper distance $L$ (measured along the radial geodesic) from the horizon (see fig. 1). We introduce two dimensionless quantities

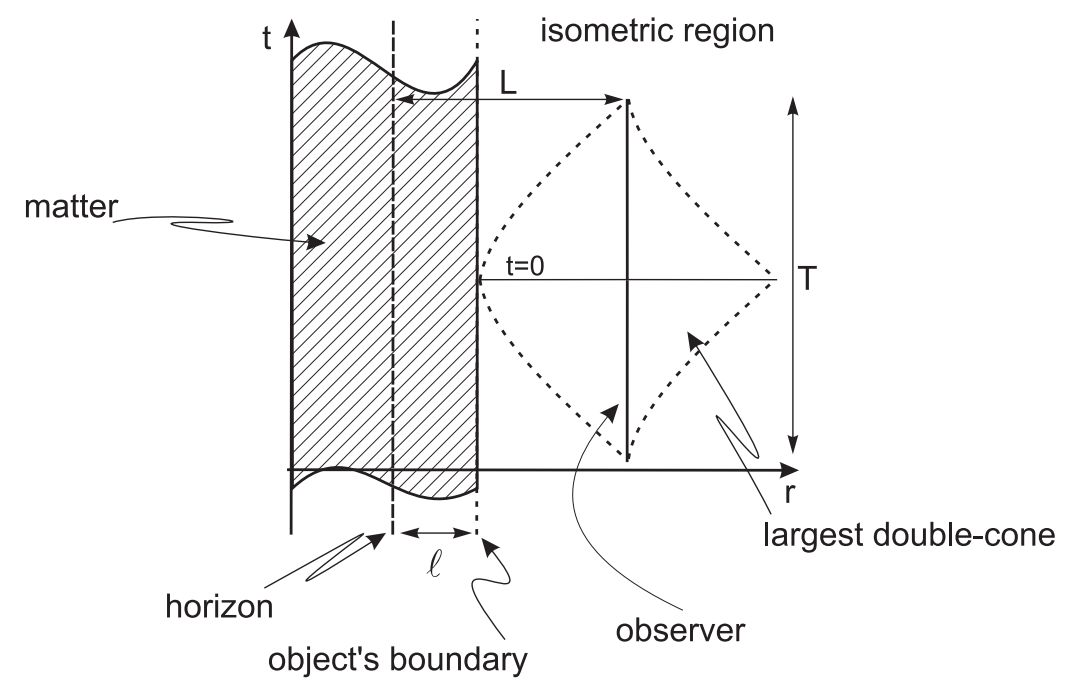

FIG. 1: Geometrical setting. The exterior part of the spacetime of a spherical object is isometric to the Schwarzschild spacetime (the object is compact).

$$
\begin{aligned}
& \epsilon_{1}=a / r_{s}-1, \\
& \epsilon_{2}=b / r_{s}-1,
\end{aligned}
$$

where $a$ is the radial coordinate of the surface of the object, and $b$ is the radial coordinate of the observer. It is a simple task to find the largest double-cone contained in the isometric region of both spacetimes. This in turn will give us the longest duration of common measurements allowed by locality arguments (see appendix $\mathbf{A}$ ). We set up a radial, outgoing null geodesic, crossing $t=0$ at $r=a$, and search for the intersection with the word-line of the observer. The longest available time of measurement, found in this way is

$$
T=2 r_{s}\left[\epsilon_{2}-\epsilon_{1}+\ln \left(\epsilon_{2} / \epsilon_{1}\right)\right]
$$

Moreover, for $\epsilon_{1}<\epsilon_{2} \ll 1$ a simple connection between $\epsilon_{1}, \epsilon_{2}$ and $\ell, L$ exists:

$$
\begin{aligned}
\epsilon_{1} & =\left(\ell / 2 r_{s}\right)^{2}, \\
\epsilon_{2} & =\left(L / 2 r_{s}\right)^{2} .
\end{aligned}
$$

For very small $\ell$ and $L$, the equation (29) still allows for a fairly long measurement times $T$ if, for instance, $\ell$ and $L$ differ by one order of magnitude. The largest double-cone, spanned 
by an observer at $L$ has a duration (measured in the global time) of

$$
T \approx 4 r_{s} \ln (L / \ell)
$$

We emphasize that there is an essential difference between $T$ derived here and (for instance) $T$ 's derived for static observers in a flat spacetime. There, if we imagine a static boundary located at $x=\ell$ and an observer at $x=L$, then the longest time of measurement for which an analogous double-cone still does not "touch" the boundary is $T=L-\ell$. Thus making $\ell$ small does not prolong $T$ very much - in contrast to the result in our setting, eq. (32).

\section{BOUNDS ON THE ENERGY DENSITY OF GROUND STATES FOR STATIC SPACETIMES OF COMPACT OBJECTS}

\section{A. Scaling and the bounds for long measurements}

For the purposes of the argument presented in this paper, it is necessary that the smearing function, $w(t)$ be of compact support. In order to investigate the limit of long measurements, we shall chose a certain function $w(t)$, and consider its normalized, scaled version:

$$
w_{\lambda}(t)=\sqrt{\lambda} w(\lambda t) .
$$

The scaling factor is chosen in such a way, that the rescaled sampling functions remain

properly normalized, $\int d t w_{\lambda}^{2}(t)=1$. Evidently, the limit of small $\lambda$ will correspond to long measurements. The rescaled $g_{\lambda}(\omega)$ will be important in what follows,

$$
g_{\lambda}(\omega)=\frac{1}{\lambda} g(\omega / \lambda) .
$$

\section{B. Bounds on the difference of energy densities}

The main aim of this paper is to compare the (known) energy density of the Boulware state $\langle\varrho(r)\rangle_{B}$ (defined on the Schwarzschild spacetime) with the unknown energy densities of ground states $\langle\varrho(r)\rangle_{G}$ for spacetimes of compact objects. The comparison will be made at the points lying in the region, where both spacetimes are described by the Schwarzschild metric.

However, two different geometries lead to inequivalent global algebras of observables, which makes it conceptually difficult to compare physical predictions, such as expectation 
values of certain global observables (eg. the total energy, cf. [14]). For purposes of this paper, we will need to compare the expectation values of a quantum observable (the energy density) in different states (which are defined on different spacetimes); it is necessary to verify, that we take the same observable in both cases (it does not suffice, for instance, if the geometry in the vicinity of the world-line of the measuring apparatus is identical, see appendix (A). A sort of comparison we need is possible only for causally complete regions with compact closure, such as double-cones of the form $\mathcal{O}=J^{+}(x) \cap J^{-}(y)$, if such regions can be isometrically embedded into both spacetimes (fig. 11). Then, the algebras of local non-linear observables $\mathcal{W}(\mathcal{O})$ associated with this region (that is, algebras of free fields, Wick polynomials, time-ordered products and energy-momentum-tensor operators smeared with test functions supported in the region $\mathcal{O}$ ) will be isomorphic 1]. This allows us to select a single local observable, the energy density smeared with a sampling function supported in $\mathcal{O}, \rho(w, \vec{x})$, and compare its expectation values in the states $B$ and $G$. Both of these states are ground states for the respective static spacetimes.

Suppose we take the QEI (21) with respect to the Boulware state $B$. It follows, that for any Hadamard state $\psi$ (on the algebra of observables on the Schwarzschild spacetime) there holds:

$$
\langle\varrho(w, \vec{x})\rangle_{\psi}-\langle\varrho(w, \vec{x})\rangle_{B} \geqslant Q_{B}[w, \vec{x}]
$$

If we now take the sampling function $w(t)$ with a compact support, such that $\varrho(w, \vec{x})$ belongs to the algebra of observables, $\mathcal{W}(\mathcal{O})$, of a double cone $\mathcal{O}$ located in the region isometric to both spacetimes, then it is sufficient if $\psi$ is a state on the algebra $\mathcal{W}(\mathcal{O})$. The restrictions of $B$ and $G$ to the region $\mathcal{O}$ are Hadamard states on $\mathcal{W}(\mathcal{O})$. Then we can develop [39] QEI with respect to either of these states. For instance, we have

$$
\langle\varrho(w, \vec{x})\rangle_{B}-\langle\varrho(w, \vec{x})\rangle_{G} \geqslant Q_{G}[w, \vec{x}] .
$$

Analogously, by changing the role of spacetimes used in the argument, we will find

$$
\langle\varrho(w, \vec{x})\rangle_{G}-\langle\varrho(w, \vec{x})\rangle_{B} \geqslant Q_{B}[w, \vec{x}]
$$

from the quantum inequality derived with respect to $B$. As the energy density of the Boulware state is known, the former of these inequalities provides an upper bound on $\langle\varrho(f, \vec{x})\rangle_{G}$, while the latter provides a lower bound. In what follows, we will investigate both of these bounds. 


\section{Bound from below}

In this section we will recall the QEI w.r.t. the Boulware state [7, 17], and show how it can be utilized to restrict the energy densities of ground states for spacetimes of compact objects. As we argue in appendix B, the QEI (26) can be employed to restrict the difference of the energy densities of the ground state $G$ and the Boulware state $B$ only if the sampling function $\omega^{2}(t)$ is such, that the smallest double cone containing the part of world-line of the static observer, for which $w^{2}(t)>0$, is contained in the region isometric with the part of the Schwarzschild spacetime (see fig. 1).

We will now review some of the properties of the operator $A$ specific to the Schwarzschild spacetime. The nature of the point $r=r_{s}$ (which is a regular singular point of the wave equation) is similar to that of $r=\infty$ in this respect: none of the generalized eigenfunctions of $A$ is locally square-integrable near $r=r_{s}$ (w.r.t. the scalar product (10)). Kay has shown [18], that $A$ defined on $\mathcal{C}_{0}^{\infty}\left(\Sigma_{t}\right)$ is essentially self-adjoint, and therefore for a selfadjoint extension the boundary conditions at $r=r_{s}$ are imposed automatically. This unique extension is such, that there remain two generalized eigenfunctions of the operator $A$ for each positive eigenvalue $\omega^{2}$. They are usually denoted in the literature by $\overrightarrow{\mathrm{R}}_{\omega l}(r)$ and $\overleftarrow{\mathrm{R}}_{\omega l}(r)$ (the former describes a wave purely outgoing to $r=\infty$, while the latter a wave purely falling through the horizon $r=r_{s}$ ). Close to the horizon, an approximate calculation due to Candelas [2] reveals:

$$
\begin{aligned}
\sum_{l=0}^{\infty}(2 l+1)\left|\overrightarrow{\mathrm{R}}_{\omega l}(r)\right|^{2} & \approx \frac{4 \omega^{2}}{\left(1-r_{s} / r\right)} \\
\sum_{l=0}^{\infty}(2 l+1)\left|\overleftarrow{\mathrm{R}}_{\omega l}(r)\right|^{2} & =\frac{1}{r_{s}^{2}} \sum_{l=0}^{\infty}(2 l+1)\left|\overleftarrow{\mathrm{B}}_{l}(\omega)\right|^{2} \\
\langle\varrho(L)\rangle_{B} & \approx \frac{1}{480 \pi^{2} L^{4}}
\end{aligned}
$$

Although the sum involving $\overleftarrow{\mathrm{R}}_{\omega l}$ cannot be evaluated analytically, Jensen, McLaughlin and Ottewill [19] have found an explicit (though approximate) expression for the transmission coefficients, $\overleftarrow{\mathrm{B}}_{l}(\omega)$, for $\left(\omega r_{s} \ll 1\right)[40]$.

$$
\overleftarrow{\mathrm{B}}_{l}(\omega) \approx \frac{(l !)^{3}}{(2 l+1) !(2 l) !}\left(-2 i \omega r_{s}\right)^{l+1}
$$

In the case of long measurements, only the leading behavior of the mode sums, for small frequencies $\omega$ will be relevant; we will, according to (39), simplify the second sum by retaining 
the $l=0$ term only,

$$
\sum_{l=0}^{\infty}(2 l+1)\left|\overleftarrow{\mathrm{R}}_{\omega l}(r)\right|^{2} \approx 4 \omega^{2}+\frac{1}{r_{s}^{2}} O\left[\left(\omega r_{s}\right)^{4}\right] .
$$

Let us turn to the QEI with respect to the Boulware state, for an observer located at the proper distance $L$ from the horizon (the radial coordinate of this observer will simply be denoted by $r$ ). We consider the re-scaled weight functions $w_{\lambda}(t)$ (see section IVA). The $Q_{B}\left[w_{\lambda}\right]$ (26) for the QEI with respect to the Boulware state, after a further substitution $p \rightarrow \lambda p, \omega \rightarrow \lambda \omega$, reads

$$
\begin{aligned}
\left|Q_{B}[w, \vec{x}]\right| \approx & \frac{1}{16 \pi^{3} \lambda} \int_{0}^{\infty} \lambda d \omega \int_{0}^{\infty} \frac{d p}{p} \\
& \left\{\frac{\lambda^{2} p^{2}}{1-r_{s} / r}+\frac{1}{4 r^{2}} \partial_{r}\left[r^{2}\left(1-r_{s} / r\right) \partial_{r}\right]\right\}\left[\frac{4 \lambda^{2} p^{2}}{1-r_{s} / r}+4 \lambda^{2} p^{2}\right] \cdot g(\omega+p) .
\end{aligned}
$$

As the above expression is intended to be the leading term of the asymptotic expansion of the exact $Q_{B}[w, \vec{x}]$ for $\lambda \rightarrow 0$ we drop all the sub-dominant terms in $\lambda$, and arrive at

$\left|Q_{B}[w, \vec{x}]\right| \approx \lambda^{2} \frac{r_{s}^{2}}{16 \pi^{3} r^{4}\left(1-r_{s} / r\right)^{2}} \int_{0}^{\infty} d \omega \int_{0}^{\infty} d p p g(\omega+p) \approx\left(\lambda r_{s}\right)^{2} \frac{I}{\pi^{3} L^{4}} \approx\left(\lambda r_{s}\right)^{2} \frac{480 I}{\pi}\left|\langle\varrho(L)\rangle_{B}\right|$,

where an approximate relation between $r$ and $L$ (similar to (30) ) was utilized, and the remaining, positive and finite double integral of $g(p+\omega)$ was denoted by $I$ :

$$
I=\int_{0}^{\infty} d \omega \int_{0}^{\infty} d p p g(\omega+p)=\frac{1}{2} \int_{0}^{\infty} d u g(u) u^{2}
$$

Altogether, the following theorem was proved:

Theorem 1. Let $G$ denote the restriction of the ground state on the compact object's spacetime to the region isometric with the Schwarzschild spacetime. The (time-independent) expectation value of the local energy density operator, evaluated at a small distance $L$ from the horizon $\langle\varrho(L)\rangle_{G}$ is bounded from below by:

$$
\langle\varrho(L)\rangle_{G}-\langle\varrho(L)\rangle_{B} \geqslant-\left(\lambda r_{s}\right)^{2} \frac{480 I}{\pi}\left|\langle\varrho(L)\rangle_{B}\right|
$$

where $\lambda$ is scale factor and $I$ is connected to $g(\omega)$ and $w(t)$ according to (41), (23). Note, that $\langle\varrho(L)\rangle_{B}$ is given by (38).

Remark: If the sampling function $w^{2}(t)$ has a support on an interval of unit length, then $\lambda=1 / T$, where $T$ is the rescaled time of measurement. The largest available $T$, (32), and 
therefore the smallest available $\lambda$, are the only quantities in (42) which depend on $\ell$, that is, on the distance from the horizon up to which both spacetimes are isometric. For given $\ell$, one can further sharpen the bound by varying over allowed sampling functions $\omega$ (i.e. by finding the minimal value of $I$, see appendix (C).

We also note, that the bound (42) can in principle be established at any distance $L$ from the horizon. However, only for small/large $L$ an explicit estimate of the mode sum is known 2], and therefore only in these cases is it possible to approximate the RHS of (42) by an explicit expression. Let us emphasize the physical meaning of (42): in the limit $\ell \rightarrow 0$ one obtains from it a strong result $\langle\varrho(L)\rangle_{G} \geqslant\langle\varrho(L)\rangle_{B}$.

\section{Remarks on the bound from above}

In order to find a bound from above on $\langle\varrho(r)\rangle_{G}$, in the region isometric with the Schwarzschild spacetime, we proceed as follows: firstly we search for a QEI w.r.t. the state $G$; this inequality allows all other states to exhibit only a limited negative energy densities (negative means: below $\langle\varrho(r)\rangle_{G}$ ). Subsequently we use the fact, that the energy density of the Boulware state is known, and according to the QEI this density cannot be much lower than $\langle\varrho(r)\rangle_{G}$. Such a reasoning provides an upper bound on $\langle\varrho(r)\rangle_{G}$.

The mode sum of the generalized eigenfunctions of the operator $A$, for the spacetime of a compact object, which is needed in order to estimate $Q_{G}[w]$ (26), will not be known, even approximately as in the case of Boulware state. Consequently, the result we shall prove here will be weaker: we will only prove, that there exists a QEI with respect to the ground state, and we will estimate how the functional $Q_{G}[w, \vec{x}]$ decays for large sampling times $T$.

To proceed, we note that as a consequence of the wave equation the functional $Q_{G}[w, \vec{x}]$ can also be written in the form:

$$
Q_{G}[w, \vec{x}] \doteq-\frac{1}{2 \pi} \int_{0}^{\infty} d \omega \int \frac{d p}{2 p} \sum_{l, m}\left[\frac{p^{2}}{f(t)}\left|F_{I}(\vec{x})\right|^{2}-\partial_{i} F_{I}(\vec{x}) \partial^{i} \overline{F_{I}}(\vec{x})\right] g(\omega+p)
$$

from which it is evident that the integrand is pointwise positive. We can now rescale the QEI, by taking the rescaled sampling functions $w_{\lambda}(t)$. We prove in the appendix $\mathbb{B}$ that $Q_{G}\left[w_{\lambda}, \vec{x}\right]$ fulfills for small $\lambda$ :

$$
Q_{G}\left[w_{\lambda}, \vec{x}\right]<\lambda c_{2} Q_{K M S}\left[w_{\lambda}, \vec{x}\right]
$$


where $Q_{K M S}\left[w_{\lambda}, \vec{x}\right]$ is the form of the right-hand-side of the QEI with a KMS state taken as the reference state. This functional is bounded for each finite $\lambda$, and approaches a finite limit for $\lambda \rightarrow 0$ (at least for spacetimes with compact Cauchy surfaces), as has been shown by Fewster and Verch [20].

Although the above estimate constitutes an upper bound on the energy density of the ground state $G$, its properties are not quite the same as that of the lower bound (eq. (42)). Not only is the assured decay property (in general) weaker, but also we have no explicit estimate (at present) of the absolute magnitude of the function $c_{2} \cdot Q_{K M S}\left[w_{\lambda}, \vec{x}\right]$. Moreover, this estimate is not uniform with respect to the changes of the internal geometry of the object (i.e. it does still depend on $\ell$ ). Consequently, even though we are able to prove the existence of an upper bound on the energy density of the ground state (which is a new result), its physical significance is weaker than that of (42) - in particular, it does not even assure, that the energy density is negative, for sufficiently small $\ell$ at fixed $L$. In order to derive a sharper bound, it appears necessary to consider particular cases of concrete spacetimes, and estimate the low-frequency behavior of the mode sums on these spacetimes. We are currently pursuing this line of thought.

\section{DISCUSSION AND OUTLOOK}

In this work we have investigated the expectation values of the energy density operator for ground states of a massless, scalar quantum field, propagating on a static, spherically symmetric spacetimes the exterior part of which is isometric to the Schwarzchild spacetime.

We would like to recall the most important, in our opinion, results of this paper: firstly, we have shown that the quantum energy inequalities supplied with arguments taking into account the necessary localization properties of observables, allow for a development of meaningful bounds on the expectation value of the energy density of a ground state, which is otherwise difficult to compute analytically. This method was originally proposed by Fewster and Pfenning in their investigation of the energy densities for ground states on flat spacetime with boundaries (Casimir setup) ([12]). Secondly, by means of a simple geometrical observation, we have shown, that these bounds are especially interesting if one considers curved-spacetime context and the exterior region of the compact object stretches up to a very small distance $\ell$ from the Schwarzschild horizon. In this case, long times of 
measurement are granted (eq. (32) ), and these make the bounds provided by QEIs especially tight. Thirdly, a concrete example of these bounds was developed, and it was shown, that the energy density of a ground state can be more negative than the energy density of the Boulware state (at the same point) only by a limited amount, with the bound depending significantly on $\ell$ (the difference falls as $1 / \ln ^{2}(\ell)$ ). Finally, we have made some preparatory steps for obtaining an upper bound on the energy density of the ground states. In particular: by recalling some abstract results on the existence of ground and KMS (thermal equilibrium) states, for a class of spacetimes fulfilling the condition (B3), we have argued, that there will exist a bound of similar nature (provided by the QEI), and we have estimated the necessary rate at which this bound "sharpens" for long times of measurement. Moreover, we have shown, that only the low-frequency (that is $\omega r_{s} \ll 1$ ) behavior of the "mode-sums" will be necessary for a development of a concrete upper bound on the energy density of the ground state. We hope to return to the issue of upper bound in a future publication.

It should be clear, that if the bound from above, the existence of which we have proved here, turned out in a more careful analysis to be of similar nature to the bound from below (established here), then the physical consequences would be profound. One could argue, by investigating ground states for a family of static spacetimes with the value of $\ell$ decreasing towards zero, that quantum field-theoretical effects necessarily become significant in this case, that is the energy density of ground states would necessarily be large in magnitude and negative (cf. [21] for a physically motivated proposal in this direction). It is precisely this type of behavior that Roman and Bergmann found [22] as indispensable in their general search for energy densities necessary for reverting the formation of trapped surfaces. Note, however, that what they found as physically implausible, namely, that the energy conditions need to be violated in the region of low density of ordinary matter, would become a necessity - as a consequence of the bound from above, should a bound with properties similar to the bound from below indeed be found.

With respect to the generalization of the results of this paper we note, that the energy density also diverges to minus infinity for a number of ground states of static spacetimes with horizons [23], notably for the static parametrization of a part of the de Sitter spacetime (which is of interest for scenarios presented in [15, 16, 21]). It appears, that our arguments can be generalized to these spacetimes as well (with the Schwarzschild spacetime replaced by another static spacetime with a horizon). We remark, that our bounds can also be employed 
as a "consistency check" for approximate or numerical computations of energy densities for spacetimes of compact objects (such as these presented in [24]): typically in such cases one has an approximate version of the two-point function as well as the energy density. The low-frequency behavior of the former can be employed in order to derive a bound, which must be satisfied by the latter.

The most far-reaching goal which we see for the type of arguments presented here would be to remove the assumption that the spacetime is static and the field resides in the ground state, and instead investigate the energy densities for spacetimes undergoing a (spherical) gravitational collapse. It would be particularly interesting to take a spacetime, which was initially static (and therefore possessed a distinguished ground state which could followed for later times), and later collapsed to a Schwarzschild black hole. For such a situation Fre-

denhagen and Haag determined in a rigorous manner [25], that the inward-looking detector (at late times and large distances from the horizon) registers the Hawking radiation. If their analysis could be adapted to detectors located close to the horizon, or equivalently - if one could argue that the state investigated by these authors approximates the Unruh vacuum state at late times, then this would give all the information needed for a quantum inequality w.r.t. the non-stationary state of quantum fields on the spacetime of a collapsing object (for the Unruh vacuum there are approximate expressions for the two-point function, as well as the energy-density [2]).

\section{Acknowledgments}

I would like to thank Chris Fewster for his critical remarks, particularly in the early stages of this work, as well as for making the results of his collaborative work with Michael Pfenning available to me prior to publication.

\section{APPENDIX A: LOCALIZATION OF NON-LINEAR OBSERVABLES FOR QFT IN CURVED SPACETIMES}

For our arguments in this paper it is essential, that there exists the largest allowed amount of time for a local common measurement made by (static) observers located at the position corresponding to the distance $L$ from the horizon. (Otherwise, the bounds provided by 
quantum inequalities - for infinite time of measurement - would imply equality of the energy densities $\langle\varrho(L)\rangle_{B}$ and $\left.\langle\varrho(L)\rangle_{G}\right)$. This restriction on the time of measurement is provided by the the necessity to fit the smallest double-cone containing the observer's world-line during the measurement to the region, where the spacetimes of interest are isometric (the exterior region). On general grounds, taking into account the hyperbolic character of the underlying wave equation, it is known that local observables (even linear fields) belong to the algebras associated with causally complete regions (see chapter III.3 of [26], and the time-slice axiom in [1]), and double cones are examples of such regions. Here we would like to point out that this localization is not only a formal requirement, but rather a physical necessity. This corroboration stems its relation to the principle of "Local Position Invariance", which is one of the forms of the equivalence principle 41]. It asserts that the outcome of any nongravitational experiment is independent of where and when in the universe it is performed; the fundamental constants of non-gravitational physics should be constants in space and time.

In experiments the LPI is verified with the help of the following procedure (see figure 2): two precise frequency standards (such as atomic clocks) are synchronized with a light signal. They follow their world-lines (which can assumed to be geodesic lines in order to rule out the influence of acceleration on the clocks) and continuously send light signals which carry the information about their states. Those signals are compared at a single event. The result is scrutinized against general relativistic predictions, namely, one calculates the geometric lengths of both world-lines. If the time lapse the atomic clocks have measured is proportional to the length of their world-lines, then indeed the non-gravitational experiments (here the quantum optical experiments) are independent of the position in the universe. Therefore, clearly, if the LPI is fulfilled then the experimental result depends only on the geometry in the region of spacetime which contains world-lines of the clocks and all the causal geodesics which join them (the clocks must be synchronized and their state must be compared). Thus, the LPI which at the moment is supported by strong experimental evidence [27] implies that the results of experiments depend on the geometry in the double-cone containing the entire measurement setup. This is precisely the localization region for observables, which we use in this paper. 


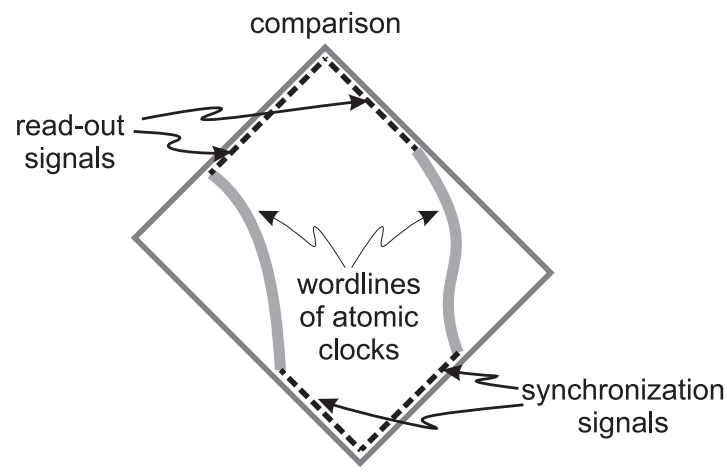

FIG. 2: GPS-like test of local position invariance. The experimental result is allowed to depend only on the gravitational field in the smallest causal normal neighborhood containing the whole measurement apparatus (together with the final signal read-out).

\section{APPENDIX B: DECAY OF QEIS FOR LARGE SAMPLING TIMES AND THE EXISTENCE OF GROUND AND THERMAL EQUILIBRIUM STATES}

In this section we will argue, that the spacetimes of (certain) compact, spherical objects admit ground and KMS (thermal equilibrium) states for massless fields. The existence of a ground state is an a priori condition for an investigation of the expectation value of the energy density operator with respect to this state, which we do throughout this paper. On the other hand, the existence of KMS states provides a general estimate of the long term behavior of the QEI, which is necessary in section IVD

In the part concerned with the existence of ground and KMS states will use the approach and the methods developed by Kay [18], and Kay and Wald [28], necessary for the construction of ground and KMS states on the Schwarzschild spacetime (exterior part of the Kruskal spacetime).

In the case of Schwarzschild spacetime Kay noted [18], that although $A$ does not have a positive lower bound (for massless fields), it fulfills

$$
\int_{\Sigma_{t}} u A u d x d \Omega \geqslant \int_{\Sigma_{t}} \alpha_{S}(x)|u|^{2} d x d \Omega
$$

for $u \in \mathcal{C}_{0}^{\infty}\left(\Sigma_{t}\right)$, with a strictly positive function $\alpha_{S}(x)$ :

$$
\alpha_{S}(x)=\frac{g \partial_{r} g}{r}=\frac{r_{s}\left(1-r_{s} / r\right)}{r^{3}},
$$

(the index $S$ indicates, that $\alpha_{S}$ is related to the Schwarzschild spacetime). This property allowed him to conclude that 
Lemma 1 (Proposition A4.9 and Theorem 4.5 of [18]). In the case of Schwarzschild spacetime, the operator $A$ is such, that

- $\mathcal{C}_{0}^{\infty}\left(\Sigma_{t}\right) \subset \mathcal{D}\left(\alpha_{S}{ }^{-1 / 2}\right) \subset \mathcal{D}\left(A^{-1 / 2}\right)$, where $\mathcal{D}\left(A^{-1 / 2}\right)$ denotes the domain of the inverse of the square root of $A$, which coincides with $\mathcal{D}\left(\mathrm{h}^{-1}\right)$.

- The one-particle structure $\left(k, \mathcal{H}, e^{i \mathrm{ht}}\right)$ is regular over the symplectic space $(S, \sigma, \mathcal{V}(t))$, $S=\mathcal{C}_{0}^{\infty}\left(\Sigma_{t}\right) \oplus \mathcal{C}_{0}^{\infty}\left(\Sigma_{t}\right)$. This means that $\mathrm{h}$ is strictly positive, and $k S \subset \mathcal{D}\left(\mathrm{h}^{-1 / 2}\right)$.

The results of Kay, quoted above, can be generalized to the case of static spherically symmetric spacetimes of (some) compact objects. For these spacetimes, let us introduce the condition

$$
\alpha(r)=\frac{g \partial_{r} g}{r}>0 \quad \forall r \in[0, \infty),
$$

which due to $g>0$ reduces to $\partial_{r} g(r)>0$. Using standard notation for spherically symmetric spacetimes generated by a perfect fluid [29],

$$
m(r)=4 \pi \int_{0}^{r} d s \rho(s) s^{2},
$$

we find that condition (B3) is equivalent to

$$
m(r)+2 \pi r^{3}[p(r)-\rho(r)]>0 .
$$

For the above condition to be fulfilled it is sufficient (although not necessary), if the spacetime is generated by a fluid of positive pressure with positive density which is a non-increasing function of $r$, [42].

For spacetimes fulfilling the condition (B3) an analogue of the theorem of Kay can be proven:

Lemma 2. For spherically symmetric, static spacetimes, whose metric is parameterized as in (3), which satisfy (B3), the conclusions of the lemma 1 hold.

Proof. The will adapt the proof of Kay [18], which was established for the Schwarzschild spacetime. The essential spacetime-specific feature which he uses, is the condition (B1) together with (B2). They are replaced here, by assumption, by the condition (B3). Apart from this, we do not see any part of the proof which would need to be altered (note, that our Cauchy surfaces $\Sigma_{t}$ are complete by assumption and therefore the standard arguments employing the well-posedness of the Cauchy problem, which imply essential self-adjointness of $A$ on $\mathcal{C}_{0}^{\infty}\left(\Sigma_{t}\right)$, can also be applied). 
With these statements at hand we may also conclude (again for spacetimes fulfilling (B3)) that the KMS (thermal equilibrium) states exist, due to the theorem of Kay and Wald:

Lemma 3 (Lemma 6.2 of [28]). If the one-particle ground state structure is regular (i.e. if the Hamiltonian $\mathrm{h}$ is strictly positive), and if

$$
k S \subset \mathcal{D}\left(\mathrm{h}^{-1 / 2}\right)
$$

then the KMS (thermal) states can be constructed for any inverse temperature $\beta \in \mathbb{R}^{+}$. The symplectic space $S$ and the operator extracting positive frequencies, $k$, are defined as in section $I I A$.

The asymptotic behavior of QEIs derived with respect to the ground state can be approximated if the KMS states on the considered spacetime exist. Let us recall the form of the KMS two-point function, smeared in both time variables (separately) with a real, compactly supported function $\chi$ :

$$
\lim _{\vec{y} \rightarrow \vec{x}} \omega_{2}^{\beta}(\chi, \vec{x}, \chi, \vec{y})=\int_{0}^{\infty} \frac{d \omega}{2 \omega} \sum_{l, m} \operatorname{coth}(\beta \omega / 2)|\hat{\chi}(\omega)|^{2}\left|F_{l m}(\omega, \vec{x})\right|^{2},
$$

By existence of KMS states we know, that the integrand is locally integrable near $\omega=0$. In chapter IV, it was necessary to estimate the long-term (that is: small $\lambda$ ) behavior of the right-hand-side, $Q_{G}\left[w_{\lambda}, \vec{x}\right]$, of quantum inequalities derived with respect to the ground state $G$, (43). Clearly it is the term with derivatives that induces the dominant behavior; we will estimate it here. For sampling functions $w(t)$ such, that $\hat{w}(p)$ is sufficiently concentrated around $p=0$ there exist constants $c_{1}$ and $c_{2}$ such that the dominant part of $Q_{G}\left[w_{\lambda}, \vec{x}\right]$ fulfills (with $\varrho=\lambda p^{\prime}, \omega=\lambda w^{\prime}$ )

$$
\begin{aligned}
& \lambda \int_{0}^{\infty} d \omega \int_{0}^{\infty} \frac{d p}{p} \sum_{l m} \frac{1}{\lambda}\left|\partial_{i} F_{l m}(\omega, \vec{x})\right|^{2} g_{\lambda}(\omega+p)= \\
&= \lambda \int_{0}^{\infty} d \omega^{\prime} \int_{0}^{\infty} \frac{d p^{\prime}}{p^{\prime}} \sum_{l m} \frac{1}{\lambda^{2}}\left|\partial_{i} F_{l m}\left(\omega^{\prime} \lambda, \vec{x}\right)\right|^{2} g\left(\omega^{\prime}+p^{\prime}\right)< \\
&< \lambda c_{1} \int_{0}^{\infty} d \omega^{\prime} \int_{0}^{\infty} \frac{d p^{\prime}}{p^{\prime}} \sum_{l m} \frac{1}{\lambda^{2} p^{\prime}}\left|\partial_{i} F_{l m}\left(\omega^{\prime} \lambda, \vec{x}\right)\right|^{2} g\left(\omega^{\prime}+p^{\prime}\right)= \\
&= \lambda c_{1} \int_{0}^{\infty} d \omega \int_{0}^{\infty} \frac{d p}{p^{2}} \sum_{l m}\left|\partial_{i} F_{l m}(\omega, \vec{x})\right|^{2} g_{\lambda}(\omega+p)< \\
&<\lambda c_{2} \int_{0}^{\infty} d \omega \int_{0}^{\infty} \frac{d p}{p} \sum_{l m} \operatorname{coth}(\beta \omega / 2)\left|\partial_{i} F_{l m}(\omega, \vec{x})\right|^{2} g_{\lambda}(\omega+p) .
\end{aligned}
$$


But the latter is just the form of $Q_{K M S}\left[w_{\lambda}, \vec{x}\right]$, multiplied with $\lambda c_{2}$, that would be obtained for a quantum energy inequality with the KMS state as the reference state: in particular it is bounded for each finite $\lambda$. It is remarkable, that the limit $\lambda \rightarrow 0$ of $Q_{K M S}\left[w_{\lambda}, \vec{x}\right]$ exists (is finite). This has been shown by Fewster and Verch in their paper on the relation between QEIs and thermodynamic equilibrium conditions, 20]. This is the conclusion of their theorem 4.7, which asserts that passive (in particular KMS) states fulfill what they call a limiting QEI. It follows, that the following limit

$$
\lim _{\lambda \rightarrow 0} \int_{0}^{\infty} d \omega \int_{0}^{\infty} \frac{d p}{p} \sum_{l m} \operatorname{coth}(\beta \omega / 2)\left|\partial_{i} F_{l m}(\omega, \vec{x})\right|^{2} g_{\lambda}(\omega+p)
$$

exists. Although we will always have a finite $\lambda$ (due to the locality requirement), this result shows that our estimate picks up the leading term of the asymptotic expansion of $Q_{G}\left[w_{\lambda}, \vec{x}\right]$. However, it should be mentioned, that Fewster and Verch have assumed compactness of the Cauchy surfaces $\Sigma_{t}$, and that this assumption appears essential to their derivation. Our

estimate of the decay of $Q_{G}\left[w_{\lambda}, \vec{x}\right]$ can be seen as a strengthening of their result for ground states, namely, it asserts that if KMS states exist on a static spacetimes with compact Cauchy surfaces, then the right-hand-side of the QEI with respect to the ground state decays at least as $\lambda^{-1}$ for large sampling times ( small $\lambda$ ).

\section{APPENDIX C: NOTE ON THE OPTIMIZATION OF QUANTUM ENERGY IN- EQUALITIES}

The lower bound on the energy density of ground states developed in this paper, (42), still contained a possibility to optimize among sampling functions $w^{2}(t)$ of compact support in the interval of unit length. More precisely, the optimization problem can be formulated as follows: one asks for a minimal value of the functional

$$
I=\frac{1}{2} \int_{0}^{\infty} d u|\hat{w}(u)|^{2} u^{2}
$$

for real functions $w(t)$, which are compactly supported in the interval $[0,1]$, with the normalization condition $\int_{0}^{1} d t|w(t)|^{2}=1$. The functions in the domain of $I$ need not to be smooth (i.e. it suffices if they lead to finite values of the functional). If we note that for symmetric functions (i.e. $w(t)=w(1-t)$ )

$$
I=\pi \int_{-\infty}^{\infty}\left|\frac{d w}{d t}\right|^{2}
$$


then the optimization problem can easily be solved, namely the functions $w(t)$ must necessarily vanish at the endpoints of the interval [43], and therefore can be expanded in the series of eigenfunctions of the (selfadjoint) operator

$$
H=-\frac{1}{2} \frac{d^{2}}{d t^{2}}
$$

with the Dirichlet boundary conditions at the endpoints. Thus the optimization problem reduces to a simple quantum mechanics: the normalization condition for $w(t)$ is easily seen as the usual normalization of wave functions, and the functional $I$ is just the expectation value of $H$ in a state described by $w(t)$. Obviously the eigenstate corresponding to the lowest eigenvalue, $w_{0}(t)=\sqrt{2} \sin (\pi t)$, minimizes such a functional. Therefore the minimal value of $I$ is $\pi^{2} / 2$. We note, that a similar argument can be used for less-trivial optimization problems turning up in the context of QEIs, namely one can apply them for functionals of the form $I_{\alpha}=\int_{0}^{\infty} d u|\hat{w}(u)|^{2} u^{\alpha}$ with $1 \leqslant \alpha \leqslant 2$, as in this case the domain of the Dirichlet extension of $H$ is dense in the domain of $I_{\alpha}$.

[1] R. Brunetti, K. Fredenhagen, and R. Verch, Commun. Math. Phys. 237, 31 (2003).

[2] P. Candelas, Phys. Rev. D 21, 2185 (1980).

[3] D. Deutsch and P. Candelas, Phys. Rev. D 20, 3063 (1979).

[4] H. Sahlmann and R. Verch, Commun. Math. Phys. 214, 705 (2000).

[5] L. H. Ford, Proc. Roy. Soc. Lond. A. 364, 227 (1978).

[6] L. H. Ford and T. A. Roman, Phys. Rev. D55, 2082 (1997).

[7] C. J. Fewster and E. Teo, Phys. Rev. D59, 104016 (1999).

[8] C. J. Fewster and E. Teo, Phys. Rev. D 59, 104016 (1999).

[9] C. J. Fewster and B. Mistry, Phys. Rev. D68, 105010 (2003).

[10] C. J. Fewster and R. Verch, Commun. Math. Phys. 225, 331 (2002).

[11] C. J. Fewster and M. J. Pfenning, J. Math. Phys. 44, 4480 (2003).

[12] C. J. Fewster, notes from a talk given at DESY, June (2003); Paper in preparation with M.Pfenning.

[13] R. M. Wald, Quantum field theory in curved spacetime and black hole thermodynamics (The University of Chicago Press, 1994).

[14] A. Herdegen, to appear in Ann. H. Poincare (2005). 
[15] P. O. Mazur and E. Mottola, Proc. Nat. Acad. Sci. 111, 9545 (2004).

[16] M. Visser and D. L. Wiltshire, Class. Quant. Grav. 21, 1135 (2004).

[17] M. J. Pfenning and L. H. Ford, Phys. Rev. D 57, 3489 (1998).

[18] B. S. Kay, Commun. Math. Phys. 100, 57 (1985).

[19] J. G. Jensen, B. P. McLaughlin and A. C. Ottewill, Phys. Rev. D 45, 3002 (1992).

[20] C. J. Fewster and R. Verch, Commun. Math. Phys. 240, 329 (2003).

[21] G. Chapline, E. Hohlfeld, R. B. Laughlin, and D. I. Santiago, Int.J.Mod.Phys. A18 (2003).

[22] T. A. Roman and P. G. Bergmann, Phys. Rev. D 28, 1265 (1983).

[23] N. D. Birrel and P. C. W. Davis, Quantum Fields in Curved Space (Cambridge University Press, Cambridge, 1982).

[24] P. R. Anderson, W. A. Hiscock, and D. A. Samuel, Phys. Rev. D 51, 4337 (1995).

[25] K. Fredenhagen and R. Haag, Commun. Math. Phys. 127, 273 (1990).

[26] R. Haag, Local Quantum Physics (Springer, 1996), 2nd ed.

[27] C. M. Will, Living Rev. Relativity 4 (2001).

[28] B. S. Kay and R. M. Wald, Phys. Rep. 207, 49 (1991).

[29] R. M. Wald, General Relativity (The University of Chicago Press, 1984).

[30] K. S. Misner, C. W. Thorne and J. A. Wheeler, Gravitation (New York: Freeman and Company, 1973).

[31] C. J. Fewster and T. A. Roman, arXiv gr-qc/0507013 (2005).

[32] C. M. Bender and S. A. Orszag, Advanced mathematical methods for scientists and engineers (Springer, 1999).

[33] A. A. Starobinski and S. M. Churilov, Sov. Phys. JETP 38, 1 (1974).

[34] This means we use the convention -++ in the classification of Misner, Thorne and Wheeler [30].

[35] This measure is not the proper volume on $\Sigma_{t}$. It is the measure derived from the Gauss's theorem relating 4D integrals of 4-divergencies of vector fields to $3 \mathrm{D}$ integrals over the Cauchy surfaces of constant $t$ (for spatially compactly supported vector fields).

[36] In 7] a more general family of functions was allowed (smooth functions, decaying as $O\left(t^{-2}\right)$ for large $t$ ). Here we will confine ourselves to functions of compact support.

[37] The sign of the integrand is positive for all $\vec{x}$ and $\omega$ and $\omega_{I}$, as can readily be verified with the help of the wave equation. 
[38] These mode sums are non-local, i.e. their value at a certain point depends on the metric on the whole Cauchy surface. In our concrete case, the propagation of waves through the matter of the object needs to be considered, even in the calculation of the sums in the exterior region.

[39] The folia induced restrictions of $G$ and $B$ to $\mathcal{W}(\mathcal{O})$ coincide 1], which means that we can always express, for instance, the expectation values w.r.t. $G$ as expectation values with respect a state, say $\psi$, described by a density matrix in the GNS Hilbert space induced by $B$. Although none of these states is a ground state for $\mathcal{W}(\mathcal{O})$, it is not necessary (QEIs can be developed with respect to arbitrary Hadamard states), as only their two-point functions enter the derivation of $Q_{B}[w, \vec{x}]$ [8] (see also the discussion of structure of QEIs in [31]).

[40] More precisely, for $r_{s} \omega \ll 1$ the generalized eigenfunctions of $A$ can be approximated with the help of the method of asymptotic matching [32] (as was discovered in 33], which yields a uniform approximation of these functions $\left(\operatorname{as}\left(r_{s} \omega\right) \rightarrow 0\right)$. A necessary condition for asymptotic matching is the existence of an intermediate region $D$, such that for all $x \in D: r_{s} \omega+1 \ll$ $x \ll \frac{l+1}{r_{s} \omega}$.

[41] The discussion of the equivalence principle (which has various ingredients) as well as its current experimental status can be found in an excellent review article by C.Will [27].

[42] Obviously, for such spacetimes $\ell$ cannot be small compared to $r_{s}$ [29].

[43] For functions which are not symmetric, the same argument applies: they do not belong to the domain of the functional $I$ because of the singularity of $d w / d t$ at the endpoints. 